\title{
Capillaroscopy - a role in modern rheumatology
}

\author{
Marek M. Chojnowski, Anna Felis-Giemza, Marzena Olesińska
}

Department of Connective Tissue Diseases, National Institute of Geriatrics, Rheumatology and Rehabilitation, Warsaw, Poland

\begin{abstract}
Capillaroscopy is a non-invasive, easy and safe diagnostic technique designed to evaluate small vessels of the microcirculation in the nailfold. It can reveal both the general architecture of capillary rows and fine details of particular vessels. The most important indications for performing capillaroscopy include differential diagnosis of primary and secondary Raynaud's phenomenon, as well as assessment of scleroderma spectrum disorders. In systemic sclerosis capillary abnormalities appear and evolve in a clearly defined sequence called the scleroderma pattern, which correlates with internal organ involvement. Capillaroscopy is also listed as a systemic sclerosis classification criterion recognized by the European League Against Rheumatism (EULAR). With digitized equipment, capillaroscopy allows for precise qualitative and quantitative evaluation of the microcirculation and is a valuable tool in the rheumatologists' daily practice.
\end{abstract}

Key words: videocapillaroscopy, systemic sclerosis, dermatomyositis.

\section{Introduction}

Capillaroscopy is a non-invasive diagnostic technique designed to evaluate small vessels of the microcirculation [1]. Nailfold capillaries were first observed in the $17^{\text {th }}$ century with primitive magnifying equipment, and in the early $19^{\text {th }}$ century the first associations between inflammation and capillary alterations were made. Beginning with the works of Maurice Raynaud's [2], research in the second half of the $19^{\text {th }}$ and first decades of the $20^{\text {th }}$ century established a direct link between capillary abnormalities and certain medical conditions. In the 1930s, interest in capillaroscopy began to decline, to rise again in the 1980s and 1990s. With the advent of modern digital equipment and evidence-based methodology, at the beginning of the $21^{\text {st }}$ century, we can witness a renaissance of the capillaroscopic technique and widespread recognition of its significance.

\section{Principles of capillaroscopy Microcirculation}

The vasculature of the microcirculation consists of the smallest blood vessels in the human body - arterioles, cap- illaries and venules. The capillaries, in turn, are formed by an arterial limb, capillary loop and venous limb. This pattern is found in every tissue except liver, spleen and bone marrow, where capillaries are replaced by sinusoids. Microcirculation's main function is capillary exchange - delivery of oxygen and nutrients to tissues and removal of carbon dioxide and waste products [3]. In a systemic disease in which vascular damage is one of the pathogenetic factors, abnormalities in capillary morphology can be observed long before the onset of clinical symptoms. In patients already diagnosed with a systemic disease, damage to the capillaries may reflect the involvement of internal organs and help determine the stage of the disease [4].

Microcirculation in capillaries is routinely evaluated within the skin of the nailfold. The entire skin abounds in capillaries; however, they run perpendicular to the skin surface, and only the tip of the loop is visible. In the nailfold, terminal rows of capillaries run parallel to the skin surface and, therefore, all morphological details and the nature of the blood flow can be examined [5].

\section{Performing capillaroscopy}

A range of optical devices can be used to perform capillaroscopic examination, for example a dermato- 
Table I. Important capillaroscopic parameters

\begin{tabular}{|ll|}
\hline Capillaroscopic parameter & Normal image \\
\hline Skin transparency and visibility & Transparent, capillaries clearly visible \\
\hline Pericapillary oedema & Absent \\
\hline Subpapillary venous plexus & Visible in up to 30\% of healthy people \\
\hline Capillary array and architecture & Straight capillaries, perpendicular to the nailfold \\
\hline Capillary morphology & U-shaped \\
\hline Capillary loop diameter & $<20 \mu m$ \\
\hline Tortuosity & Usually absent \\
\hline Dilated (20-50 $\mu \mathrm{m})$ and giant $(>50 \mu \mathrm{m})$ loops & Absent \\
\hline Ramified capillaries & Absent \\
\hline Neoangiogenesis & Absent \\
\hline Haemorrhages, hemosiderin deposits & Usually absent, may be present after local trauma \\
\hline Capillary density & 9 -13 in 1 linear millimetre \\
\hline Avascular areas (distance between 2 loops $>500 \mu \mathrm{m})$ & Absent \\
\hline Capillary blood flow & Dynamic, no stasis or thrombosis \\
\hline
\end{tabular}

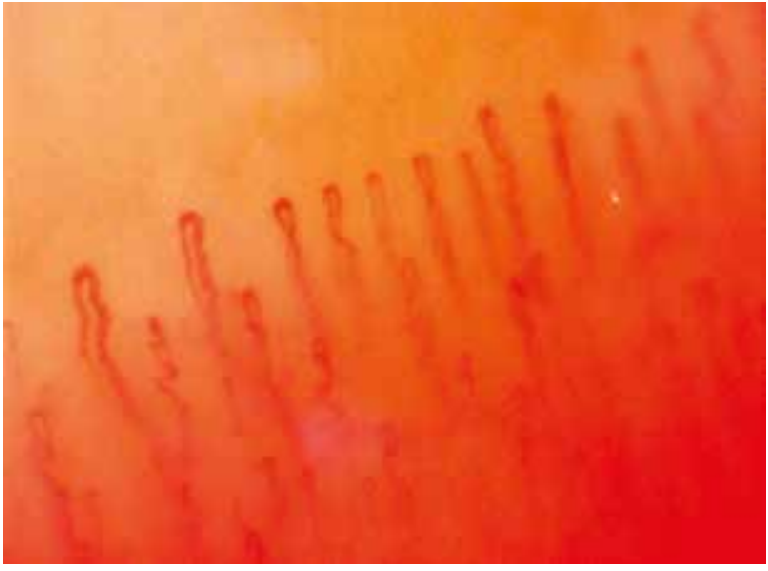

Fig. 1. Normal capillaroscopic image with U-shaped capillaries.

scope, an ophthalmoscope or a traditional microscope. However, it is best to perform the examination with equipment dedicated for capillaroscopy, i.e. a stereomicroscope or a digital videocapillaroscope. Of these two, the latter is preferred, as a hand-held probe can be easily used in every situation, e.g. for bedside examination or in patients with severe flexion contractures.

In order to enhance skin transparency, a drop of immersion oil is applied to the nailfold before capillaroscopy. In a routine examination, all fingers except the thumbs are evaluated. Each finger should be examined in two magnifications: $\times 50$, showing the general architecture of the terminal capillary row, and $\times 200-300$, in which morphological details of a single capillary can be assessed. Digital equipment allows the obtained images to be stored and used for an objective comparison if the patient needs subsequent examinations [6].

\section{Patient preparation}

Environmental factors can cause physiological constriction of capillaries, thus greatly affecting the capillaroscopic image. Before the examination, patients should be acclimatised at a temperature of $20-22^{\circ} \mathrm{C}$ for 15-20 minutes, and should refrain from smoking and drinking caffeine for 4 hours. Capillaroscopy should not be performed if the patient has recently (in the last 3 weeks) undergone any cosmetic procedure involving the nailfold area, since the consequent micro-traumas can give false-positive results [6].

\section{Important capillaroscopic parameters and normal capillary image}

There are several parameters which should be evaluated during capillaroscopy (Table I) [1, 7, 8]. A normal capillaroscopic image of a healthy control is shown in Figure 1.

\section{Raynaud's phenomenon}

The most important indication for capillaroscopy is Raynaud's phenomenon (RP) [8]. This condition usually presents in the fingers or toes, more rarely in the nose and ears, and involves a sequence of skin colour changes, attributed to vasospasm and subsequent vessel dilation. These include skin turning white (vasoconstriction), then blue (hypoxia) and finally red (reperfusion). Raynaud's phenomenon can be primary (idiopathic) or 
secondary to numerous conditions. Capillaroscopy plays a pivotal role in differentiating one from another [9].

In primary RP capillaries of the nailfold are normal, with no apparent abnormalities. It should be stressed that diagnosis of the primary condition cannot be based solely on capillaroscopy; it also requires normal levels of acute phase reactants and absence of any other clinical symptoms. It is recommended to perform capillaroscopy every $12-24$ months in primary RP, since up to $10 \%$ of these patients will develop a connective tissue disease, sometimes after decades.

In RP secondary to non-rheumatic conditions, the capillaroscopic image may be normal or present nonspecific alterations. On the other hand, in connective tissue diseases, especially in scleroderma spectrum disorders, microangiopathy of the peripheral microcirculation is prominent, with the presence of specific patterns that can be attributed to particular diseases [10].

\section{Scleroderma spectrum disorders}

Scleroderma spectrum disorders are a heterogeneous group of connective tissue diseases linked to systemic sclerosis (SSc) and exhibiting characteristic capillaroscopic abnormalities [11]. These include giant capillaries, loss of capillaries with areas of avascularisation, ramified capillaries with pathological neoangiogenesis and severe derangement of capillary architecture. All of these pathologies may be present to a different extent in scleroderma spectrum disorders. In SSc, however, they appear and evolve in a clearly defined sequence called the scleroderma pattern.

\section{Systemic sclerosis}

Systemic sclerosis (SSc) is a rare connective tissue disease presenting with diffuse fibrosis and dysfunction of internal organs due to microangiopathy [12]. In well over $95 \%$ of SSc patients, peripheral microangiopathy follows a typical scleroderma pattern, consisting of 'early', 'active' and 'late' phases (Table II, Fig. 2) [13]. Differentiation of these patterns is of great clinical significance, since the 'early' pattern can be detected many years before full clinical manifestation of SSc, and progression to 'active' and 'late' patterns closely corresponds to internal organ involvement [14]. The importance of capillaroscopy is underlined by its inclusion in the current EULAR classification criteria of SSc [15].

Capillaroscopy can be useful in predicting development of finger ulcerations in SSc patients. The capillaroscopic skin ulceration risk index (CSURI) is a tool devised by Sebastiani et al., based on the formula $D \times M / N^{2}$ ( $D$ - diameter of the biggest giant loop, $M-$ number of giant loops, $\mathrm{N}$ - number of all loops) (Fig. 3 )
[16]. In a prospective clinical trial, CSURI correctly predicted formation of new ulcerations within 3 months following capillaroscopy, with a positive predictive value of $81 \%$ for CSURI $>2.96$ and a negative predictive value of $93 \%$ for CSURI $<2.96$ [17].

\section{Dermatomyositis and antisynthetase syndrome}

Dermatomyositis (DM) and its variant, antisynthetase syndrome, are connective tissue diseases with prominent muscle and skin involvement $[18,19]$. Despite diverse clinical presentations, they share to some extent a common pathogenesis with SSc, i.e. primary damage to microcirculation. Presence of severe capillary abnormalities such as giant loops and ramified capillaries with intense neoangiogenesis is common in DM patients, with a typical scleroderma pattern occurring in up to $40 \%$ of cases [20]. Contrary to SSc, in DM the connection between the capillary image and internal organ involvement has not yet been fully studied. However, a good response to treatment and, later on, remission of the disease are often accompanied by normalization of the capillary image [21]. Capillaroscopy can be very useful in differentiating DM and polymyositis (PM). In PM, the suggested pathogenetic mechanism is an autoimmune response of cytotoxic $T$ cells, not microangiopathy as postulated in DM. Capillaroscopic findings support this theory, since in PM patients capillary abnormalities are rare, mild and comparable to the healthy population [20].

\section{Mixed connective tissue disease}

Mixed connective tissue disease (MCTD) is a clinical entity that combines symptoms of SSc, DM, rheumatoid arthritis (RA) and systemic lupus erythematosus (SLE) [22]. The incidence and extent of capillary involvement in MCTD vary greatly, from a normal image through mild

Table II. Scleroderma pattern [13]

\begin{tabular}{|c|c|}
\hline $\begin{array}{l}\text { 'Early' } \\
\text { pattern }\end{array}$ & $\begin{array}{l}\text { Preserved capillary architecture } \\
\text { Few giant capillaries and microhaemorrhages } \\
\text { No evident capillary loss }\end{array}$ \\
\hline $\begin{array}{l}\text { 'Active' } \\
\text { pattern }\end{array}$ & $\begin{array}{l}\text { Slight disorganisation of capillary architecture } \\
\text { Numerous giant capillaries and microhaemor- } \\
\text { rhages } \\
\text { Moderate capillary loss } \\
\text { Few ramified capillaries }\end{array}$ \\
\hline $\begin{array}{l}\text { 'Late' } \\
\text { pattern }\end{array}$ & $\begin{array}{l}\text { Severe derangement of capillary architecture } \\
\text { Almost absent giant capillaries and microhaem- } \\
\text { orrhages } \\
\text { Significant capillary loss with avascularisation } \\
\text { Numerous ramified capillaries with neoangio- } \\
\text { genesis }\end{array}$ \\
\hline
\end{tabular}


A
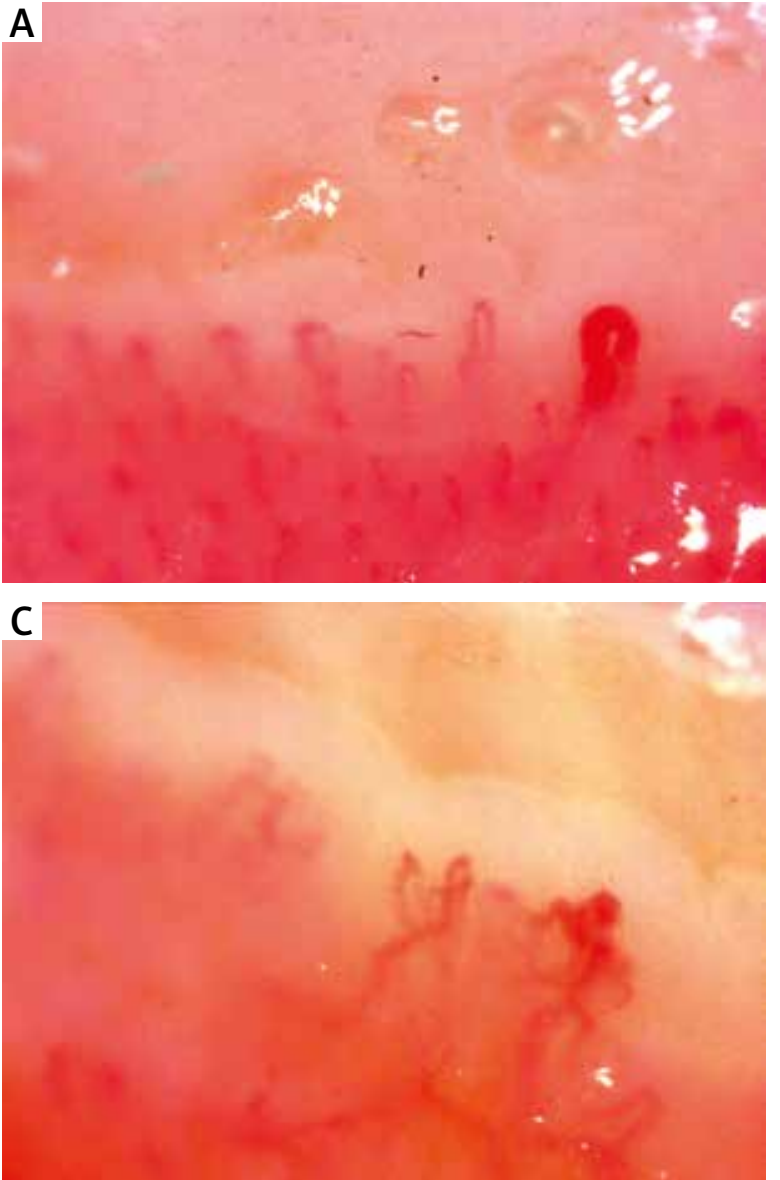

B

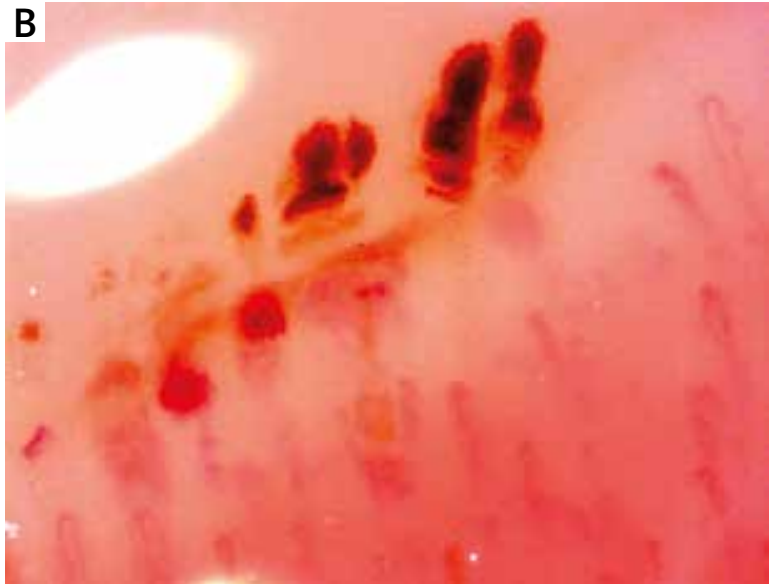

D

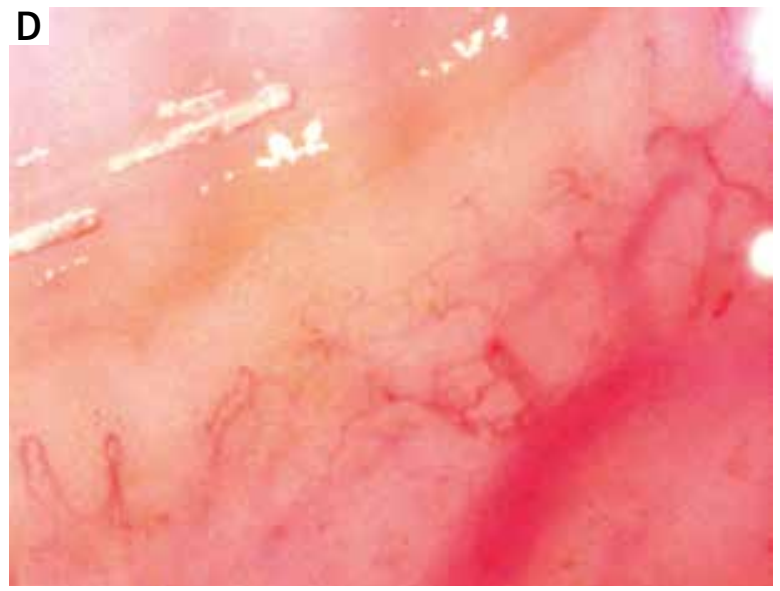

Fig. 2. Scleroderma pattern. Early (A), active (B) and late (C, D) patterns.

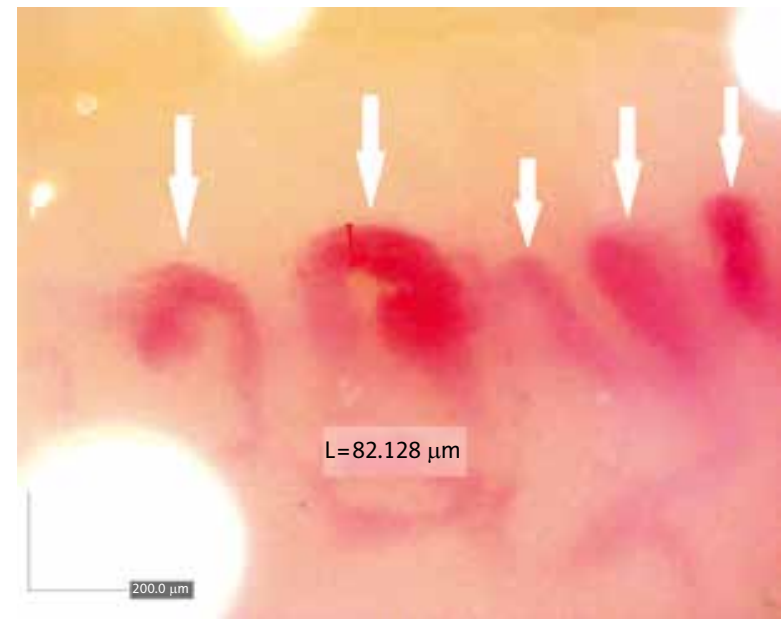

Fig. 3. Calculating CSURI: number of giant capillaries ( $M$, arrows) -5 ; diameter of the biggest loop (D) $-82 \mu \mathrm{m}$; total number of capillaries $(\mathrm{N})$ - 5. CSURI $\left(D \times M / N^{2}\right)=16.4$, which indicates high risk of new digital ulcerations. non-specific abnormalities to severe microangiopathy, rarely with a scleroderma pattern. Scleroderma-like abnormalities in MCTD often (76\% of patients) accompany interstitial lung disease (ILD) [23].

\section{Scleromyositis}

Scleromyositis (SCM) is an overlap syndrome combining features of SSC and DM/PM, associated with the presence of anti-PM/Scl antibodies. The clinical course of this disease is usually more benign than SSc, with a normal capillary image. In more severe cases, however, alterations of nailfold capillaries, especially presence of the scleroderma pattern, may predict emergence of symptoms typical of systemic sclerosis, e.g. ILD [24].

\section{Primary biliary cirrhosis}

Primary biliary cirrhosis (PBC) is a chronic, autoimmune liver disease, characterized by progressive destruction of bile ducts, cholestasis and presence of antimitochondrial antibodies (AMA). Incidence of PBC and SSC is strongly associated, with PBC being the most common 
liver disease in SSc patients. Capillaroscopy is a potential screening method in PBC patients with symptoms suggesting SSc. Similarly to ScM, scleroderma-like capillary abnormalities may correlate with ILD [25].

\section{Other connective tissue diseases}

Extensive research has been performed on capillary alterations in other connective tissue diseases, but no specific patterns have been found so far. In SLE, nailfold capillaries are often normal, and mild changes occur in up to $30 \%$ of patients. The most frequent alterations include increased tortuosity, elongated loops, and meandering bizarre loops. These abnormalities are often associated with discoid lesions on the skin of the fingers. Patients with RA are reported to have increased capillary tortuosity and prominent, clearly visible subpapillary venous plexus. Antiphospholipid syndrome (APS) and small-vessel vasculitides can both present as multiple microhaemorrhages and hemosiderin deposits, with no morphological changes in capillaries. However, it should be noted that such an image can be seen even after a minor trauma, so caution is advised when reporting capillaroscopic findings in patients with APS or vasculitis [26-28].

\section{Interstitial lung disease}

Interstitial lung disease (ILD) commonly accompanies scleroderma spectrum disorders, but can also be associated with other rheumatic diseases, infection, environmental factors, or appear with no obvious cause [29]. Capillaroscopy may be useful in equivocal cases, since patients with ILD and scleroderma-like capillary abnormalities are likely to have or soon develop SSc or an associated disease [30].

\section{Limitations of capillaroscopy}

It should be duly noted that apart from connective tissue diseases, more common medical conditions may be a cause of peripheral microangiopathy. In the course of both diabetes and hypertension, severe capillary alterations can be observed, with heterogeneously enlarged loops, capillary loss and neoangiogenesis. A pathological capillary image may also result from prolonged exposure to vibration, chemical agents, ionizing radiation and other occupational factors.

Wide field capillaroscopy performed with a stereomicroscope can have low reproducibility, due to qualitative and subjective assessment. On the other hand, videocapillaroscopy requires skill and precision to perform properly, since even small pressure of the probe on the nailfold can disrupt capillary blood flow and influence the study results.
Table III. Indications for capillaroscopy

\begin{tabular}{|l|}
\hline $\begin{array}{l}\text { Differential diagnosis of primary and secondary Raynaud's } \\
\text { phenomenon }\end{array}$ \\
\hline Staging of systemic sclerosis \\
\hline $\begin{array}{l}\text { Predicting formation of new digital ulcers in systemic } \\
\text { sclerosis (CSURI) }\end{array}$ \\
\hline Response assessment of dermatomyositis treatment \\
\hline Differential diagnosis of dermatomyositis and polymyositis \\
\hline Screening for interstitial lung disease in MCTD, ScM and PBC \\
\hline Differential diagnosis of interstitial lung disease \\
\hline
\end{tabular}

\section{Conclusions and summary of clinical applications}

Capillaroscopy is an easy and safe method for diagnosing pathologies of peripheral microcirculation. Its applications are summarized in Table III. With digitized equipment, capillaroscopy allows for precise qualitative and quantitative evaluation of the microcirculation and is a valuable tool in the rheumatologists' daily practice.

The authors declare no conflict of interest. This paper is part of the first author's PhD thesis.

\section{References}

1. Tavakol ME, Fatemi A, Karbalaie A. Nailfold Capillaroscopy in Rheumatic Diseases: Which Parameters Should Be Evaluated? Biomed Res Int 2015; 2015: 974530.

2. Raynaud M. De l'asphyxie locale et de la gangrène symétrique des extrémités. Leclerc, Paris 1862.

3. Granger DN, Senchenkova E. Inflammation and the Microcirculation. Morgan \& Claypool Life Sciences, San Rafael 2010.

4. Herrick AL, Cutolo M. Clinical implications from capillaroscopic analysis in patients with Raynaud's phenomenon and systemic sclerosis. Arthritis Rheum 2010; 62: 2595-2604.

5. Lin KM, Cheng TT, Chen CJ, et al. Clinical Applications of Nailfold Capillaroscopy in Different Rheumatic Diseases. J Intern Med Taiwan 2009; 20: 238-247.

6. Smith V, Pizzorni C. The Videocapillaroscopic Technique. In: Cutolo M. Atlas of Capillaroscopy in Rheumatic Diseases. Elsevier, Milan 2010: 25-31.

7. Allen J. Capillaroscopy in Healthy Subjects of Different Ages. In: Cutolo M. Atlas of Capillaroscopy in Rheumatic Diseases. Elsevier, Milan 2010: 49-53.

8. Cutolo M, Grassi W, Matucci Cerinic M. Raynaud's phenomenon and the role of capillaroscopy. Arthritis Rheum 2003; 48: 3023-3030.

9. Kuryliszyn-Moskal A, Kita J, Hryniewicz A. Raynaud's phenomenon: new aspects of pathogenesis and the role of nailfold videocapillaroscopy. Reumatologia 2015; 53: 87-93.

10. Bukhari M, Hollis S, Moore T, et al. Quantitation of microcirculatory abnormalities in patients with primary Raynaud's 
phenomenon and systemic sclerosis by video capillaroscopy. Rheumatology (Oxford) 2000; 39: 506-512.

11. Maricq HR, Valter I. A working classification of scleroderma spectrum disorders: a proposal and the results of testing on a sample of patients. Clin Exp Rheumatol 2004; 22 (3 Suppl 33): S5-S13.

12. Elhai M, Avouac J, Kahan A. Systemic sclerosis: Recent insights. Joint Bone Spine 2015; 82: 148-153.

13. Cutolo M, Sulli A, Pizzorni C, et al. Nailfold videocapillaroscopy assessment of microvascular damage in systemic sclerosis. J Rheumatol 2000; 27: 155160.

14. Cutolo M. Capillaroscopy links to clinical pathological issues in systemic sclerosis. In: Cutolo M. Atlas of Capillaroscopy in Rheumatic Diseases. Elsevier, Milan 2010: 173-179.

15. Van den Hoogen F, Khanna D, Fransen J. 2013 classification criteria for systemic sclerosis: an American College of Rheumatology/European League against Rheumatism collaborative initiative. Arthritis Rheum 2013; 65: 2737-2747.

16. Sebastiani M, Manfredi A, Colaci M, et al. Capillaroscopic skin ulcer risk index: a new prognostic tool for digital skin ulcer development in systemic sclerosis patients. Arthritis Rheum 2009; 61: 688-694.

17. Sebastiani M, Manfredi A, Vukatana G, et al. Predictive role of capillaroscopic skin ulcer risk index in systemic sclerosis: a multicentre validation study. Ann Rheum Dis 2012; 71: 6770

18. Mahil S, Marks D, McCormack M, et al. Dermatomyositis. Br J Hosp Med (Lond) 2012; 73: C18-22.

19. Imbert-Masseau A, Hamidou M, Agard C. Antisynthetase syndrome. Joint BoneSpine 2003; 70: 161-168.

20. Manfredi A, Sebastiani M, Cassone G, et al. Nailfold capillaroscopic changes in dermatomyositis and polymyositis. Clin Rheumatol 2015; 34: 279-284.

21. De Angelis R. Capillaroscopy in dermatomyositis. In: Cutolo M. Atlas of Capillaroscopy in Rheumatic Diseases. Elsevier, Milan 2010: 115-120.

22. Tani C, Carli L, Vagnani S, et al. The diagnosis and classification of mixed connective tissue disease. J Autoimmun 2014; 48-49: 46-49.

23. de Holanda Mafaldo Diógenes A, Bonfá E, Fuller R, et al. Capillaroscopy is a dynamic process in mixed connective tissue disease. Lupus 2007; 16: 254-258.

24. Guillen-Del Castillo A, Pilar Simeón-Aznar C, Fonollosa-Pla V, et al. Good outcome of interstitial lung disease in patients with scleroderma associated to anti-PM/Scl antibody. Semin Arthritis Rheum 2014; 44: 331-337.

25. Rigamonti C, Bogdanos DP, Mytilinaiou MG, et al. Primary Biliary Cirrhosis Associated with Systemic Sclerosis: Diagnostic and Clinical Challenges. Int J Rheumatol 2011; 2011: 976427.

26. Ingegnoli F. Capillaroscopy in Systemic Lupus Erythematosus. In: Cutolo M. Atlas of Capillaroscopy in Rheumatic Diseases. Elsevier, Milan 2010; 131-140.

27. Sulli A. Capillaroscopy in Antiphospholipid Syndrome. In: $\mathrm{Cu}$ tolo M. Atlas of Capillaroscopy in Rheumatic Diseases. Elsevier, Milan 2010; 141-144.

28. Hermann W. Capillaroscopy in Rheumatoid Arthritis and Sjögren's Syndrome. In: Cutolo M. Atlas of Capillaroscopy in Rheumatic Diseases. Elsevier, Milan 2010; 161-168.
29. Fischer A, du Bois R. Interstitial lung disease in connective tissue disorders. Lancet 2012; 380 (9842): 689-698.

30. Antoniou KM, Margaritopoulos G, Economidou F, et al. Pivotal clinical dilemmas in collagen vascular diseases associated with interstitial lung involvement. Eur Respir J 2009; 33: 882896. 